\title{
Power Saving Mechanisms for Ad hoc networks Based on Handshaking Information Tapping
}

\author{
Abdelfettah Belghith and Wafa Akkari \\ HANA Research Group \\ National School of Computer Sciences (ENSI) \\ University of Manouba, Tunisia 2010 \\ abdelfattah.belghith@ensi.rnu.tn \\ wefa.akkari@gmail.com
}

\begin{abstract}
This paper presents an optimization of the functional properties of IEEE 802.11 PSM to improve its energy conservation without changing or requiring additional control or management frames. PSM relies on a handshaking procedure to announce data traffic before its transmission. This announcement traffic puts a heavy constraint on the sizes of both the ATIM window and the beacon interval and consequently on the network throughput, delay and power saving. All Stations are required to stay awake during the entire ATIM window, and hence they can infer from the already exchanged handshaking frames whether other stations in their vicinities have decided to stay active during the rest of the beacon internal. As such, many announcements could be avoided.

First, we present the Neighborhood Aware Power Saving Mechanism (NA-PSM) that reduces the announcement period and hence provides much throughput and power saving than PSM, yet reduces the mean sojourn time of data frames. Second, we present a further refinement of NA-PSM based on the fact that a station having no more traffic to send or receive can power down its tranceiver even though it has already issued an ATIM frame or an ATIM-ACK frame. We show using extensive simulations that the proposed mechanisms outperform clearly PSM in terms of throughput, delay and power saving.
\end{abstract}

Keywords: Power Conservation, Ad-Hoc networks, PSM(Power Saving Mode), J-Sim simulator, TA-PSM(Traffic Aware Power Saving Mode), Performance Evaluation

\section{INTRODUCTION}

The last few decades have seen a tremendous growth in wireless communication. Wireless Local Area Networks WLANs stand out among the most successful technologies in supporting both user mobility and high data rates. Two different operational modes are defined in IEEE 802.11: the infrastructure networks in which a specific central entity manages communications between stations, and the ad hoc networks where spontaneous mobile stations communicate with each other over multiple wireless hops [1]. In ad hoc networks, nodes (also referred interchangeably as stations) are mobile and rely solely on their portable battery energy. Therefore, energy conservation mechanisms are needed to expand the lifetime of the different nodes and the whole network [2]. Various techniques, in hardware and software, have been proposed to ensure energy conservation for mobile nodes in WLANs [1], [2], [3], [4], [5], [6], [7] , [8], [9], [10], [11], [12], [13], [14] , [15], [19]. IEEE 802.11 defined a Power Saving Mechanism (PSM) for both infrastructure networks (BSS: Basic Service Set) and ad hoc networks (IBSS: Independent Basic Service Set) [1]. PSM in IEEE 802.11 is basically based on powering down node's wireless interfaces whenever deemed possible (no traffic activity). PSM for Ad-Hoc networks divides time into specific periods called beacon intervals. At the beginning of each beacon interval, a starting sub-period called the ATIM window is reserved during which all nodes must be active to exchange synchronization frames (beacons) and to announce buffered data frames (ATIM messages). Each node buffers temporally data frames to be relayed to a neighbouring node whose transceiver is powered down. These data frames will be announced during the ATIM window of the following beacon interval. Any node having sent or received announcements must remain active during the entire beacon interval. Newly generated data frames are transmitted by the generating node during the current beacon interval only if this node has announced buffered data frames to the destined nodes, otherwise such traffic should be 
delayed to be first announced in the next ATIM window. It is interesting to notice that a station must announce all its buffered traffic to all destined neighbours regardless whether they intend or not to remain active in the beyond ATIM window (whether or not they have already been informed to do so by other stations). Moreover, traffic forwarding to a neighbour station during the current beacon interval is only possible if the station has announced buffered data frames to the destined nodes, otherwise such traffic should be delayed to be first announced in the next ATIM window. This functional behaviour inherent to IEEE 802.11 PSM results not only in an energy waste which could be avoided for better power conservation efficiency but also in an increase in the sojourn time of data frames and some throttling of the network throughput.. In this paper, we first explicit the functional properties of PSM for ad hoc networks and then propose ways to improve its mechanism, without requiring, however, any change in IEEE 802.11 frame formats nor additional control or management frames. Basically, an adequate power conservation protocol should save energy at its maximum at light traffic conditions since most of the nodes could power down their transceivers, yet it should deliver as much traffic as possible at high traffic loads. An energy conservation algorithm should in no way conserve energy by delaying or throttling traffic from flowing towards its destination.

We first propose a neighborhood aware power saving mechanism NA-PSM that induces the neighborhood information from the announcements exchanged between nodes during ATIM windows. This, as will be showed later, will reduce the number of ATIM announcement frames and hence allows more bandwidth to carry the data traffic, yet it will make it possible to transmit newly generated traffic and to forward traffic faster. NA-PSM allows nodes to abandon their ATIM transmissions if the destined neighbor node of this ATIM is known to stay active. NA-PSM, however, has still the shortage, as in PSM, that nodes having traffic to transmit stay active during the whole beacon interval even after finishing the transmission and the reception of all their traffic. This amounts to some power consumption which could be avoided by powering down the transceivers of these nodes immediately upon the termination of their transmission and reception. In [20], the authors proved via extensive simulations that doing so yields a substantial power saving. Thus, we propose a further refinement to our protocol called the Neighborhood and Traffic Aware Power Saving Mechanism (NTA-PSM) which adapts NA-PSM to traffic load in order to reduce the energy consumed in ad-Hoc networks without degrading the throughput or increasing the delay. The proposed NTA-PSM includes all the benefits of NA-PSM, yet allows nodes having no traffic to send, to receive or to forward to power down their transceivers.

The rest of the paper is organized as follows. In section (2), we present an overview of the power saving mechanism in IEEE 802.11 for Ad-Hoc networks (PSM). We devote section (3) to present the shortcomings of PSM. Our approach, NA-PSM (Neighborhood Aware Power Saving Mechanism) is introduced in section (4). Section (5) presents our experimental performance evaluations of the new approach and its comparison to PSM. Section (6) explains the inherited shortage within NA-PSM and presents NTA-PSM (Neighborhood and Traffic Aware Power Saving Mechanism). We confirm the contribution of our proposition through simulations using the Jsim simulator. Section (7) provides some concluding remarks.

\section{IEEE 802.11 POWER SAVING MECHANISM (PSM)}

\subsection{Synchronization in an ad hoc network}

Synchronization in an Ad-Hoc network is based on a distributed Timing Synchronization Function (TSF) that keeps the timers of all stations of the IBSS synchronized. Each node maintains a local TSF timer. Nodes expect to receive beacons every Beacon interval. A node sending a beacon sets the Beacon's timestamp equals to its own local TSF time. The beacon interval of the IBSS is established by the station that initiated the IBSS. A series of TBTTs (Theoretical Beacon Transmission Times) are defined which are exactly a beacon interval time units apart. A node shall transmit a beacon according to the following procedure:

- Awakes just before the end of the TBTT to be able to receive beacons. If the node is already in the Awake state, it suspends decrementing the back off timer for any pending non beacon or non ATIM transmission.

- Calculates a random delay upon which it transmits a beacon.

- Decrements the random delay according to the back off algorithm.

- Sends its own beacon if no beacon was received and the random delay has expired.

- Suspends the decrementing of the random delay and renouncing on its beacon if a beacon was received before the random delay has expired.

Each node adopts the timing received from any beacon that has a TSF value later than its own TSF timer. It is readily known that the IEEE 802.11TSF based mechanism works properly when the network is a single hop and fully connected. In the case of a multi-hop network, it does not function as intended [21]. In this paper, we assume a 


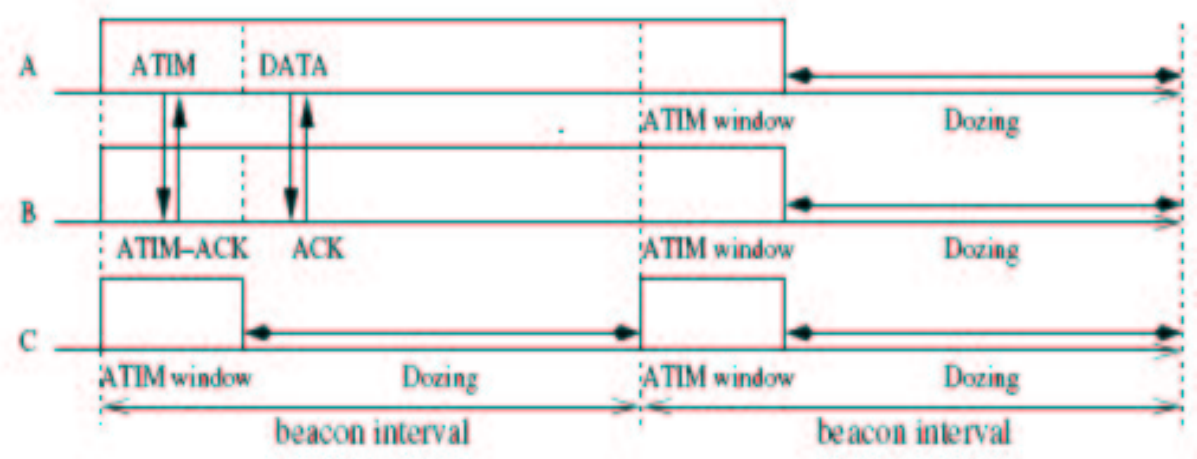

FIGURE 1: Power management in an IBSS

TSF based synchronization as defined in IEEE 802.11 [1]. Our primary goal here is rather to present our proposed power saving mechanisms NA-PSM and NTA-PSM. The issue of appropriate synchronization mechanisms for ad hoc networks is out of the scope of the current paper.

\subsection{Power management in an ad hoc network}

PSM defines two different power states:

- Awake: the station wireless interface is powered on and is able to transmit and receive. In the awake state, a station can be transmitting (station referred to be in the transmit state), receiving (station referred to be in the receive state or just idle (station referred to be in the idle state).

- Doze: the station wireless interface is power down and hence it could neither transmit nor receive (station referred to be in the Doze state).

Two management modes are defined:

- AM (Active Mode): the station is permanently in the awake state.

- PS (Power Saving): the station can be either in the Awake state or in the Doze state.

At the start of each beacon interval, each station must stay awake during a fixed time period, called the ATIM (Adhoc Traffic Indication Message) window. A station temporarily buffers data frames destined to other stations in the Doze state. These buffered data frames are announced during the ATIM window using Unicast ATIM frames. Upon reception of an ATIM frame, a station replies (immediately after just a Short Inter Frame Spacing SIFS) by sending an ATIM-ACK and stays awake for the entire beacon interval waiting for the announced frames to be received. If a station has neither sent an ATIM frame nor received an ATIM-ACK frame, it enters the Doze state at the end of the ATIM window. Announced frames are transmitted after the ATIM window using the normal CSMA/CA back off procedure. A station having sent at least one ATIM frame shall stay awake during the entire current beacon interval. In figure 1 below, station A sends an ATIM frame to station B during the ATIM window. B responds by sending an ATIM-ACK. Both stations stay awake during the entire beacon interval. Station C hasn't received or sent any ATIM frame so it enters the doze state at the end of the ATIM window.

\section{POWER AND THROUGHPUT INEFFICIENCIES OF PSM FOR AD HOC NETWORKS}

According to PSM, each node, having data frames to transmit, must announce its traffic by sending unicast ATIM frames to the designated stations during the ATIM window. Recall that an ATIM frame contains both the source and the destined station's addresses [1]. The destined stations, upon receiving the ATIM frames, reply by sending unicast ATIM-ACKs. Recall that an ATIM-ACK contains only the ATIM-ACK receiving station address [1] that is the address of the station that has sent the ATIM-ACK. Therefore, during the ATIM window, there will be ATIM and ATIM-ACK frames sent as many as there are stations having traffic to send or forward. In a an ad hoc network like the one represented in figure 2 below, there will be 10 ATIM frames and 10 ATIM-ACK frames to exchange during the current ATIM window. This means that, in a dense network with many stations having traffic to send, we need to have a 


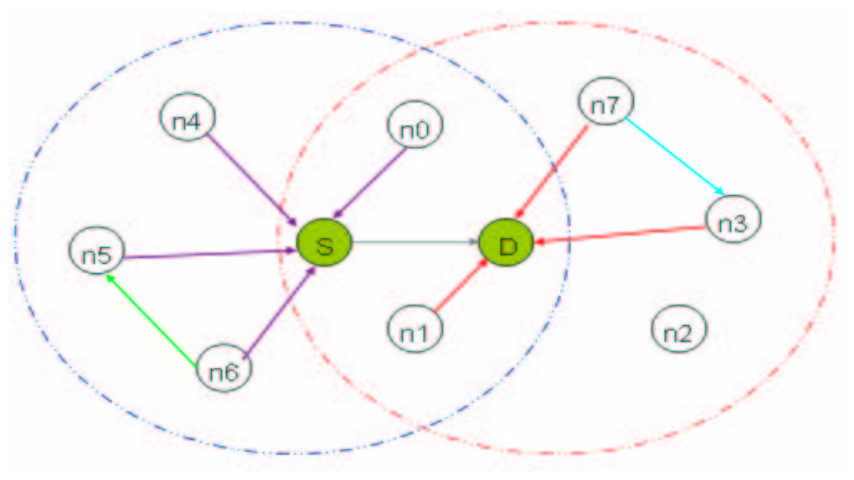

FIGURE 2: Architecture of the network

large period of announcements (ATIM window) for stations to be able to send their ATIM frames and receive the corresponding ATIM-ACK frames. However, for a given size of the beacon interval, increasing the ATIM window sub period amounts to decreasing the beyond ATIM window and so the time reserved to the data transmission is reduced. This throttles clearly the network throughput. We may think of enlarging instead the size of the beacon interval, but this amounts necessarily to an increase in the sojourn time of the data frames.

It is, however, interesting to notice that if node $S$ sends an ATIM frame destined to node D, all stations n0, n1, n4, $\mathrm{n} 5, \mathrm{n} 6$ and $\mathrm{D}$ hear this ATIM frame and consequently they know that station $\mathrm{S}$ will remain active for the beyond-ATIM window once it receives the corresponding ATIM-ACK. These neighbors of station $S$ can use this information and refrain from sending their ATIM frames since $S$ will be active anyway. This can reduce the number of exchanged ATIM and ATIM-ACK frames during the ATIM window and lead to a substantial decrease of this period of announcement (the ATIM period). The idea here is to use the information about neighbors, which is induced from the exchange of announcement frames, to reduce the ATIM window and consequently increase the throughput and decrease the delay, yet decrease also the power consumption per delivered data frame.

\section{THE NEIGHBORHOOD AWARE-POWER SAVING MECHANISM : NA-PSM}

Each station in the network maintains a data structure, hereafter referred as the Active Neighbor Table ANT, to contain the identities of the neighboring stations known to remain active beyond the current ATIM window (during the current beacon interval). This table is to be initialized to empty at the beginning of each beacon interval. The ANT is updated as follows:

- When a station hears an ATIM frame transmission not destined to itself and addressed to a node that is not already in its ANT, it inserts the source address of this ATIM frame into its ANT. The destination address of the ATIM frame is temporary saved.

- After a SIFS, this station either hears the corresponding ATIM-ACK (which happens just after a SIFS [1]) and then it inserts the saved address in its ANT, or it does not hear the corresponding ATIM-ACK and then it discards the saved address.

When a source station has traffic to deliver to a destined neighboring node and before sending an ATIM frame, it consults its ANT to see whether this destined node is already awake. If it is indeed (the destined node is in its ANT), the source does not send an ATIM-ACK but rather waits for the end of the ATIM window to transmit its data frames according to the CSMA/CA algorithm. If, however, the destined node is not in the source station ANT, the later must send its own ATIM frame to announce this traffic. In figure 3, we provide a state diagram which governs the operation of the NA-PSM for each station in the network.

1. : upon the start of a frame reception

2. : upon the end of a frame reception

3. : upon the end of a frame transmission

4. : upon the start of a frame transmission

5. : just before the TBTT time ends (the beginning of the next Beaconinterval)

6. : upon the end of the ATIM window, if the station has neither sent an ATIM frame nor an ATIM-ACK frame and has no traffic to deliver to an already awake station (by inspecting its ANT without sending an ATIM frame) 


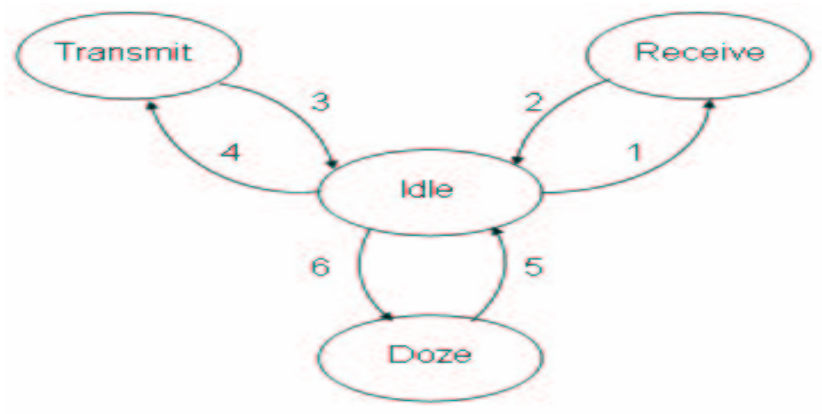

FIGURE 3: State Diagram showing the possible states and transitions for NA-PSM

By reducing the number of exchanged announcement packets (ATIM and ATIM-ACK), NA-PSM allows the use of a smaller ATIM window. This can be considered as an important advantage. In fact, when we reduce the ATIM window period, we enlarge the period reserved to data transmission and then increase the network aggregate goodput. As a consequence of the increase of the goodput, the delay of the received packets is reduced. To show how well our proposed protocol works under a small ATIM window period, we will simulate traffic with 11 sender-receiver pairs and a variable value for ATIM window.

\section{NA-PSM PERFORMANCE EVALUATION}

NA-PSM can substantially deliver more traffic and save more energy with a reduced ATIM window. To show this advantage, we have implemented NA-PSM in the Jsim simulator and compare it to PSM using different scenarios. We note here that Jsim already implements IEEE 802.11 IBSS [16], [17], [18]. We use four metrics to evaluate the proposed scheme:

- Number of delivered data frames: represents the total number of data frames delivered to a chosen destination station at the end of the simulation; namely the throughput at this destination station. This metric is useful to verify whether power saving mechanisms degrade throughput or not.

- Mean Sojourn Time: represents the average time a data frame spent in the network from its generation at the source station to its delivery to the destination station.

- Power consumption: representing the total energy consumed by all stations during the simulation time.

- Power consumed per delivered data frame: represents the total energy consumed divided by the number of delivered data frames; namely the aggregate energy goodput.

Each simulation run is performed for 500 seconds. We used CBR traffic models with different data rates, a fixed data frame size of 512 bytes, a wireless channel bit rate of $2 \mathrm{Mbps}$. The energy consumption model used throughout the simulation is defined as follows:

- Energy consumption rate in transmit state equals 0.660 Watt per second.

- Energy consumption rate in receive state equals 0.395 Watt per second.

- Energy consumption rate in idle state equals 0.296 Watt per second.

- Energy consumption rate in doze state equals 0.0 Watt per second.

We consider a beacon interval of 0.1 seconds which is the value specified for PSM in [1]. We assume an initial energy equal to 1000 Watt per station. For this scenario, we use a network composed of 14 stations as shown in figure 4 above.

Wireless connectivity for node $\mathrm{n} 3$ and $\mathrm{n} 4$ are represented by the corresponding dashed ovals. We shall pay a particular attention to the traffic flow between source station $\mathrm{n} 3$ and destination station $\mathrm{n} 4$. Data traffic between the other stations is represented by arrows. Each arrow represents a traffic load of 50 packets per second (pkt/sec) where the arrow points toward the destination station of the traffic. We start our performance evaluation by fixing the traffic load between $\mathrm{n} 3$ and $\mathrm{n} 4$ to $50 \mathrm{pkt} / \mathrm{sec}$ and we vary the ATIM window period from 0.001 seconds to 0.02 seconds. Figure 5(a) shows the number of delivered data frames from $\mathrm{n} 3$ to $\mathrm{n} 4$ as a function of the ATIM window size. 


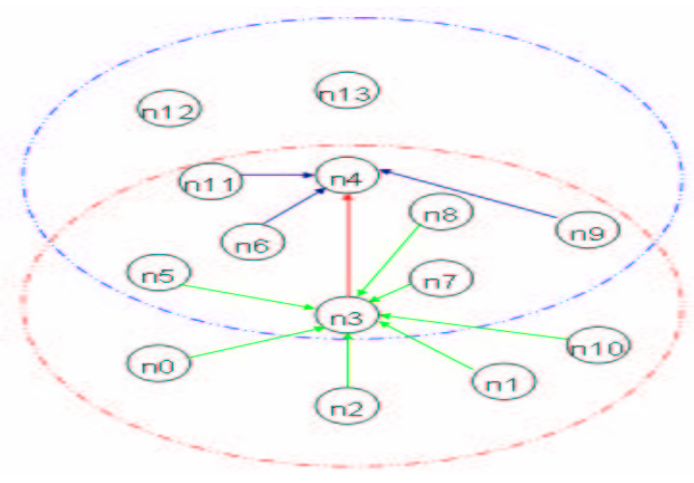

FIGURE 4: Architecture of the network

We clearly see that NA-PSM delivers more throughput than PSM for reduced ATIM window (less than 0.01 seconds). For an ATIM window size greater or equal to 0.01 seconds both NA-PSM and PSM delivers the same throughput. For our network example, it is easy to see that at most 2 ATIM frames should be transmitted during the ATIM window under the NA-PSM strategy. Indeed, if it happens that $\mathrm{n} 3$ is the first to send its ATIM frame then only one ATIM frame and its corresponding ATIM-ACK frame are needed, otherwise two ATIM frames and their corresponding ATIM-ACK are sent. However, when using PSM for the same network example, exactly 11 ATIM frames and their corresponding ATIM-ACK frames must be exchanged each ATIM window. When the ATIM window becomes smaller, PSM cannot exchange the 22 announcement frames and therefore it will throttle the throughput of the network (some data frames could not be transmitted since they could not be announced). NA-PSM, on the contrary, needs only to send a very small number of announcement frames and hence all data frames could be transmitted.

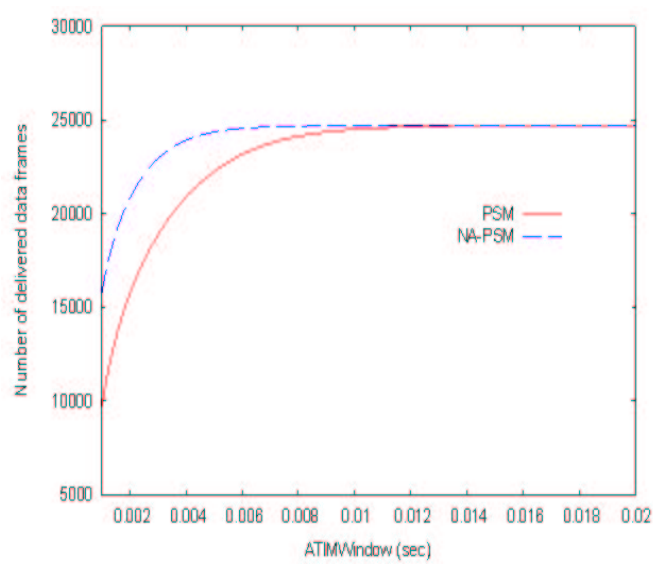

(a)

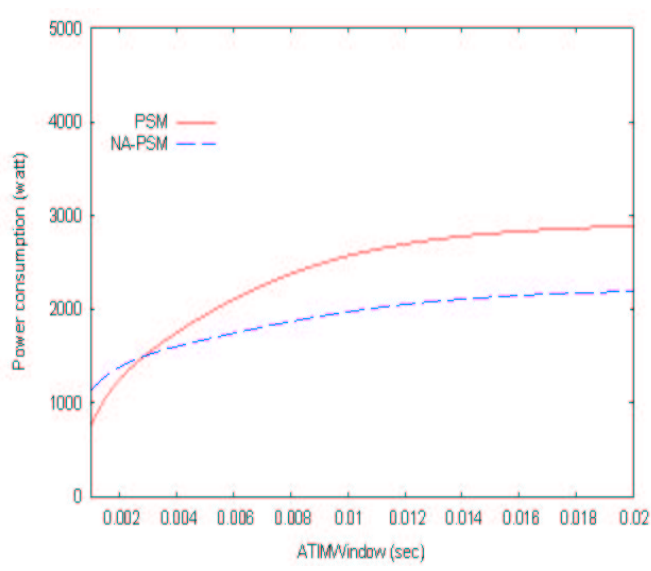

(b)

FIGURE 5: (a)(b): Number of delivered data frames and Power consumption versus ATIM window.

In figure 5(b) above, we show the power consumption as a function of the ATIM window size. We observe that NAPSM consumes much less energy than PSM when ATIM window is higher than 0.03 seconds. We notice also that the energy consumed by NA-PSM is slightly more important than the energy consumed by PSM when ATIM window is less than 0.03 seconds. Indeed, for very small values of the ATIM window, NA-PSM delivers much more traffic than PSM as portrayed on figure 5(a). Consequently, it is more informative to portray the power consumption per delivered data frame which we show in figure 6 below.

We note that NA-PSM outperforms PSM for all the values of the ATIM window. NA-PSM guarantees higher throughput than PSM, yet it provides much better energy conservation and a better power consumption per delivered data frame. Next, we will try to enhance further our proposed NA-PSM in terms of power conservation. 


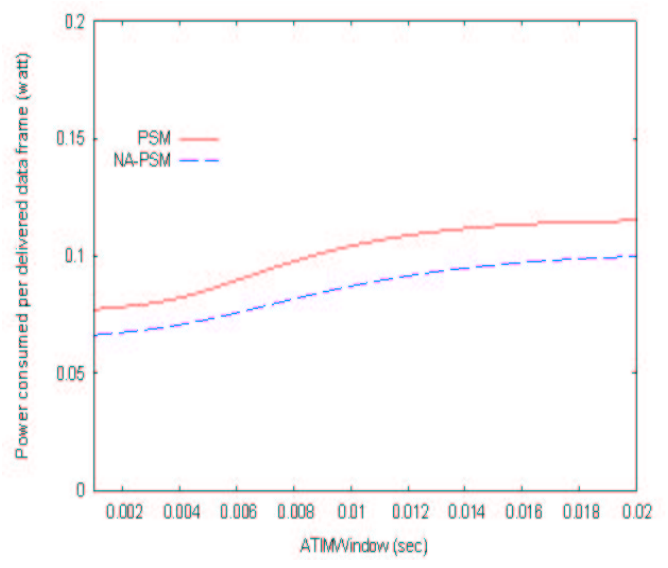

FIGURE 6: Power consumed per delivered data frame vs ATIM window

\section{THE NEIGHBORHOOD AND TRAFFIC AWARE-POWER SAVING MECHANISM : NTA-PSM}

In [1], PSM specifies that a station having sent or received an announcement or having sent a beacon must remain awake during the entire current beacon interval. In [20], the authors showed that this may lead to wasting energy especially at light traffic loads where stations should conserve the most. They propose an enhancement called the Traffic Aware Power Saving Mechanism (TA-PSM) to solve this problem by allowing nodes to enter the doze state when they are not involved in data delivery even if they have already sent a beacon, an ATIM or an ATIM-ACK. TAPSM consists on reducing the energy consumption by making PSM more sensitive to the current traffic. First, they added a one bit MoreData field (one bit) into the frame header to indicate to the receiving station that further pending data frames are buffered. If the MoreData bit is set in the received frame, both transmitting and receiving stations stay awake; otherwise, they can switch to the doze state. This allows the sending station to engage transmitting further data frames arriving within the same beacon interval and destined for this same receiving node. Secondly, a station sending a Beacon during the ATIM window enters the doze state if it has neither sent nor received ATIM frames during the current ATIM window. They showed that TA-PSM provide a much better energy conservation and a lower power consumption per delivered data frame.

In NA-PSM, a station announces its buffered data frames using Unicast ATIM frames during the ATIM window. A station receiving a unicast ATIM frame responds during the current ATIM window by sending a unicast ATIM acknowledgment. Both these stations must stay Awake during the entire beacon interval. All other stations having traffic to deliver to the source or the destination of any ATIM frame must remain Awake during the current Beacon interval. If one of these stations has just one (few) frame(s) to transmit to another station, both stations will remain Awake for the entire Beacon interval. Consequently, much more energy is consumed than needed. The idea on which NTA-PSM is founded stems from the fact that energy saving is more effective for light traffic that in heavy traffic conditions. Indeed, when the network load is light the majority of mobile nodes are able to enter the Doze state. Whenever, the load is high, mobile nodes are obliged to handle such traffic and hardly find time to go dozing. In other words, an adequate power saving approach should achieve a maximum energy saving at light network loads and should rather not throttle traffic from entering or leaving the network and from being forwarded at high traffic loads.

Our scheme consists on reducing the energy consumption by making NA-PSM more sensitive to the traffic load in the same way TA-PSM does. First, we add a MoreData field (one bit) into the frame header to indicate that further pending frames are buffered for the destination. When trying to apply the idea of TA-PSM to NA-PSM, we noticed that we can't use it fully. In fact, TA-PSM stipulates that:

- Before transmitting a frame, a station checks whether further frames are pending for the same neighbouring station. If so, the MoreData bit is set, otherwise the current frame is sent with a MoreData bit unset.

- A station receiving a data frame with the MoreData bit set must stay awake until it gets, from the same station, a frame with the MoreData bit unset.

- A station transmitting a data frame with the field MoreData set must remain awake.

- A station receiving a frame with MoreData unset sends an ACK for this frame and enters to the doze state if no more frames are due from other stations. 
- A station sending a frame with MoreData unset must wait for this frame ACK to enter the doze state if no more frames are due from and to other stations.

- At the end of the ATIM window, a station having no traffic to send or to receive enters the doze state for the rest of the beacon, even if it has sent a beacon.

n contrast, with NA-PSM, a station having finished the transmission of its buffered data frames cannot simply switch to the Doze state because other stations may rely on it (it could be marked in the ANT of another station). However, all stations but the ones that send an ATIM frame or an ATIM-ACK are able to enter the doze state upon finishing the transmission of their data frames (the station has already sent a data frame with a MoreData set and received its ACK). To evaluate our NTA-PSM and compare it to TA-PSM, PSM and to TA-PSM, we consider again the network example depicted on figure 4. The disruptive traffic loads are now 20pkt/sec each. We focus rather on of the flow sent from station $\mathrm{n} 3$ to station $\mathrm{n} 4$. We fix the ATIM window to 0.045 seconds which privileges the NA-PSM according to the curves presented in Figures 5(a) and 6(b).

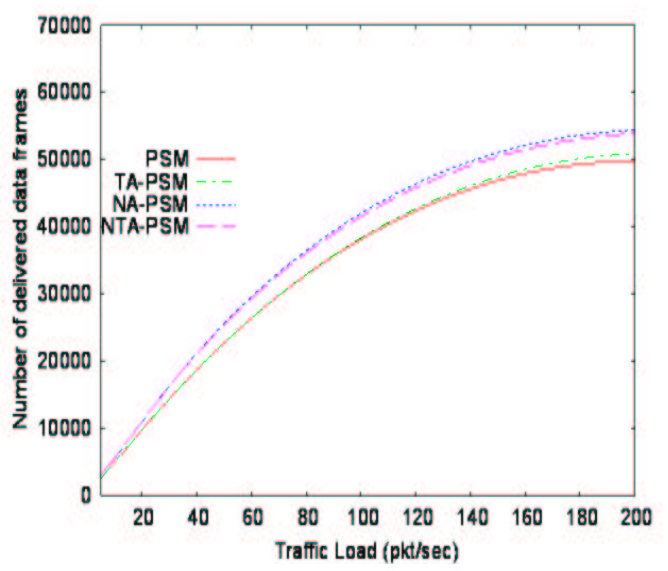

(a)

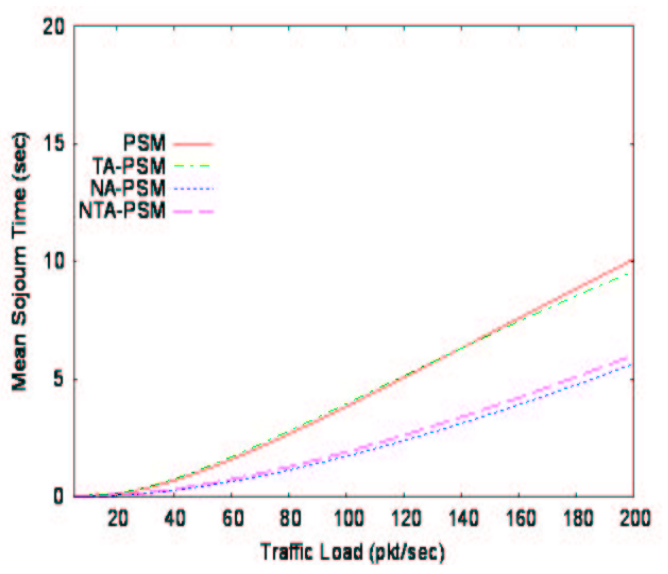

(b)

FIGURE 7: (a)(b): Number of delivered data frames and Mean Sojourn time versus traffic load.

Figure 7(a) above portrays the number of delivered data frames to station $\mathrm{n} 4$ as a function of the load traffic generated at station n3. The difference between PSM and TA-PSM is already explained in [20]. We notice that NA-PSM ensures higher throughput than PSM which can be justified by the choice of small period ATIM window (only 0.045 seconds). Both NA-PSM and NTA-PSM provide virtually the same throughput for all traffic load values. In fact these two protocols have the same functional behavior during the ATIM window.

We remarkably notice on figure 7 (b) above the gain in the mean sojourn time for the traffic delivered at node $n 4$. As mentioned in [20], TA-PSM has latency comparable to PSM. We observe that NTA-PSM reduces the mean Sojourn time nearly to the same level as NA-PSM. However, the delay induced by these mechanisms is much lower than the one induced by PSM and TA-PSM. This stems from the blocking of the traffic when using PSM or TA-PSM with such a small ATIM window size. For all buffered packets that cannot be sent, the delay within PSM or TA-PSM accumulates until the packet is received by the destination.

During the beyond ATIM window period and for NTA-PSM, nodes n0, n1, n2, n5, n6, n7, n8, n9, n10 and n11 can switch to Doze state after ending their data transmission to $n 3$ and n4. For NA-PSM all these nodes remain Awake. This explains that these protocols have the same throughput but different power consumption as shown in figures ?? and ??. We observe that NTA-PSM outperforms clearly NA-PSM for all traffic loads. This is because, for the used network example, all stations have to deliver or receive a data frame. NTA-PSM allows the stations to get into the Doze state if there is no traffic to send or to receive whereas in NA-PSM they remain active.

One thing noticeable about the power consumption curves in figure $8(a)$ is that our proposed NTA-PSM has the lowest energy level for all traffic loads. In fact, NTA-PSM owes this reduced energy consumption to the combination of two properties having each and separately proved its saving of power: the comparison between TA-PSM and PSM proved that TA-PSM is more power-conservative than PSM. In the same way, we have proved in section 6 that the power consumption relative to NA-PSM is much less than PSM. Figure $8(b)$ plots the power consumption per 


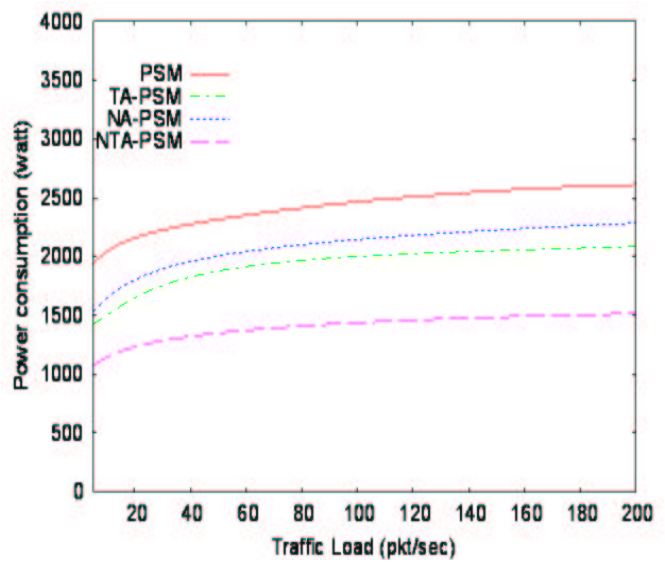

(a)

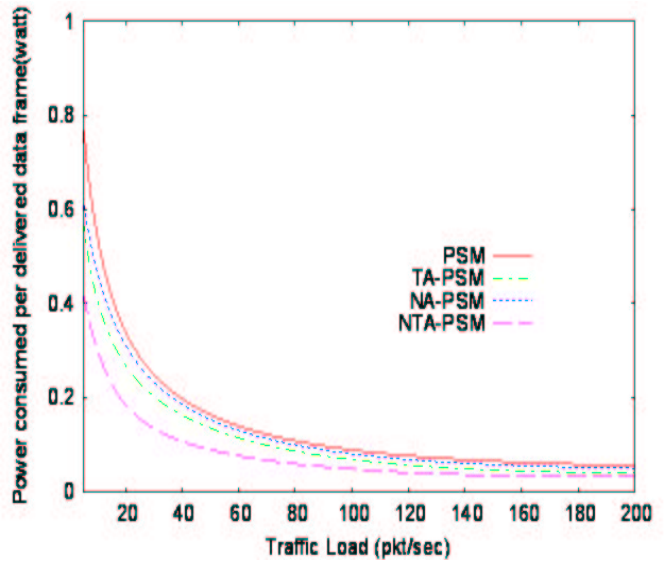

(b)

FIGURE 8: (a)(b): Power consumption and Power consumed per delivered data frame versus traffic load.

delivered data frame to $\mathrm{n} 4$ as a function of the traffic load generated by $\mathrm{n} 3$. We note that NTA-PSM outperforms all other mechanisms for all traffic loads.

To bring out the advantages of our proposed NTA-PSM, we measure the aggregate throughput and delay of all traffics in the network. We plot the total number of delivered data frames to all destinations as a function of traffic load generated at $n 3$ (figure $9(a)$ ).

Here again, NA-PSM and TA-PSM ensure the same throughput since they succeed in sending the announcement frames. We notice that these two mechanisms guarantee a gain of about $40 \%$ compared to PSM and TA-PSM in terms of total throughput. For the mean Sojourn time relative to all traffics represented in figure $9(\mathrm{a})$, we clearly see that the gain in total delay realised by NA-PSM and NTA-PSM exceeded 90

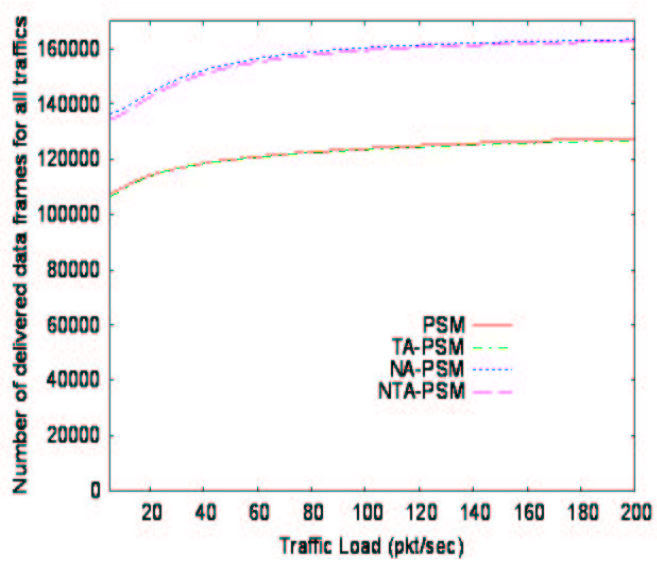

(a)

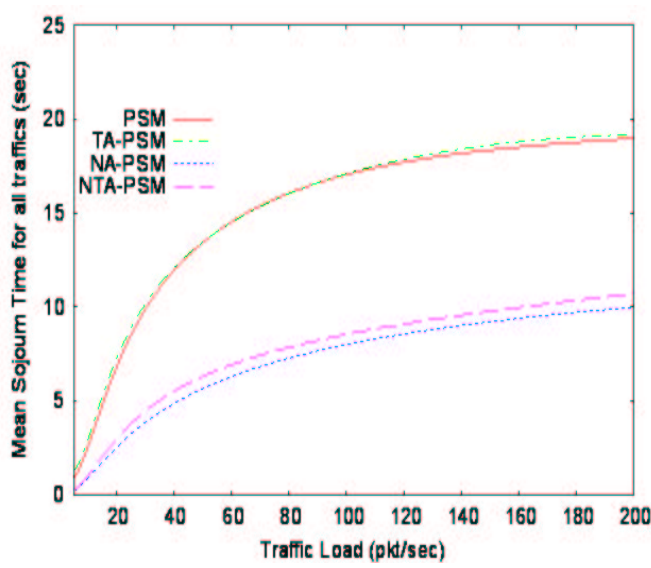

(b)

FIGURE 9: (a)(b): Aggregate number of delivered data frames and Sojourn time of cumulative traffics versus traffic load.

NTA-PSM outperforms PSM, TA-PSM and NA-PSM in terms of throughput, delay and power conservation. NTA-PSM guarantees the same throughput as NA-PSM, yet it provides better energy saving and a lesser power consumption per delivered data frame.

\section{CONCLUSION}

In this paper, we showed by means of extensive simulations that IEEE 802.11 PSM for ad hoc networks leads to certain inefficiencies with respect to the energy consumed per data frame delivered, throughput and sojourn times. These inefficiencies stem from the inherent operational behaviour of PSM. At light traffic loads where energy saving 
should be the largest, PSM obliges stations having sent a beacon or exchanged ATIM management frames to remain in the active state for the rest of the entire current beacon interval regardless the lack of further pending data frames. The huge number of announcement data frames required to be exchanged during the ATIM window period constitutes the main pitfall of PSM. This leads to an increase of the power consumption and a decrease of the throughput at the expenses of a larger mean sojourn time.

We then proposed a way to reduce substantially the number of ATIMs sent during the ATIM window by inducing the activity states of the neighbouring stations. This yielded a new mechanism called the NA-PSM. We showed using extensive simulations that NA-PSM outperforms PSM in terms of more power saving, less power consumed per delivered data frame and better throughput, yet provides much lower mean sojourn times.

We finally combined the efficiencies of both TA-PSM and NA-PSM to obtain the Neighbourhood and Traffic Aware PSM (NTA-PSM). We proved through extensive simulations that NTA-PSM improves the performance of NA-PSM, yet outperforms clearly PSM and TA-PSM in providing much less power consumption, $40 \%$ gain in throughput and $90 \%$ gain in the mean sojourn time for the example of network used. More investigations are under way to better ascertain the impact of some features of ad hoc networks such as mobility, multi hop and synchronization.

\section{REFERENCES}

[1] ANSI/IEEE Standard 802.11. (1999) Wireless LAN Medium, Access Control(MAC) and Physical, Layer (PHY) Specifications.

[2] C.E. Jones, K. Sivalingam, p. Agrawal and J Chen.(2001)A survey of energy efficient network protocols for wireless networks. ACM Journal Wireless Networks 7 (4), pp. 343-358.

[3] B. Chen, K. Jamieson, H. Balakrishman and R. Morris.(July, 2001) Span: An energy-efficient coordination algorithm for topology maintenance in ad hoc wireless networks. in MOBICOM 2001.

[4] E.S. Jung and N.H. Vaidya.(June 23-27 2002) An energy efficient MAC protocol for wireless LANs.INFOCOM 2002, New York, USA.

[5] T. Simunic and S. Boyd.(2002)Managing power consumption in networks on ships. ACM DATE.

[6] T. Zhang, P. Gurung, E. Van Den Berg, S. Madhani and A. Muttreja.(2006)Silent networking for energy constrained nodes. in press in Computer Communications, Elsevier.

[7] H. Zhu and G. Cao.(March 7-11, 2004)A power-aware and QoS-aware service model on wireless networks. IEEE INFOCOM'04, Hong Kong.

[8] J.R. Jiang, Y.C. Tseng C.S. Hsu and T.H. Lai.(2005)Quorum-based asynchronous power-saving protocols for $802.11 \mathrm{ad}$ hoc networks. ACM mobile networking and applications (MONET), special issue on algorithmic solutions for wireless, mobile, ad hoc and sensor networks, vol. 10, No. , pp. 169-181.

[9] A. Sheth and R. Han.(May 19-22, 2003)Adaptive power control and selective radio activation for low-power infrastructure-mode 802.11 LANs. Proceedings of 23rd International Conference on Distributed Computing Systems (ICDCSW'03), Providence, Rode Island, USA, pp. 812-817.

[10] M. Anand, E. Nightingale and J. Flinn.(September 14-19, 2003) Self tuning wireless network power management. Proceedings of the 9th ACM Annual International Conference on Mobile Computing and Networking (MobiCom 2003), San Diego, California, USA.

[11] R. Zheng and R. Kravets.(March 2003)On-demand Power Management for Ad Hoc Networks. Proc. of IEEE INFOCOMM 2003, San Francisco.

[12] B. Awerbuch, D. Holmer and H. Rubens.(March 7-11, 2004)The pulse protocol: energy efficient infrastructure access. IEEE INFOCOM'04, Hong Kong.

[13] H. Idoudi, M. Molnar and A. Belghith.( July 18-20, 2005)Power Aware Alternation : a novel power saving mechanism for ad hoc networks., International Conference on Performance Modeling and Evaluation of Heterogeneous Networks, HET-NET'05, West Yorkshire, U.K.

[14] J. Zhu; C. Qiao and X. Wang.(March 7-11, 2004)A comprehensive minimum energy routing scheme for wireless ad hoc networks. IEEE INFOCOM'04, Hong Kong.

[15] D.Y. Kim and C.H. Choi.(May 25-28, 2004)Adaptive power management for IEEE 802.11 based ad hoc networks. in World Wireless Congress, San Francisco,USA.

[16] J-Sim simulator.(last visited in 23-05-2005) on-line at www.j-sim.org.

[17] R. S. Nair.(March 1997)JSIM: A Java-Based Query Driven Simulation and Animation Environment. Masters Thesis (M.S. in CS Degree).

[18] W. Akkari, A. Belghith and H. Idoudi.(2006) Evaluation de l'implmentation de PSM (Power Saving Mode) sous le simulateur J-Sim . GEl'06. 
[19] Changsu Suh, Young-Bae Ko and Jai-Hoon Kim.(2005)Enhanced Power Saving for IEEE 802.11 WLAN with Dynamic Slot Allocation. LNCS, Vol. 3794, pp. 466-477.

[20] A. Belghith and W. Akkari.(2007) Traffic Aware MAC protocol in Ad-Hoc networks. The Journal of Networks, Volume 2, Issue 4, pp. 1-13.

[21] L. Huang and T. lai.(June,2002)On the scalability of the IEEE 802.11 Ad HOC Networks. In the proceedings of the ACM MOBIHOC. 